\title{
Superficies Nanoestructuradas de la Aleación Ti6Al4V. Influencia del Tiempo de Electropulido
}

\author{
Caroline Pigatto $^{(1)}$, Leonardo M. Antonini ${ }^{(1)}$, Eduardo L. Schneider ${ }^{(2)}$ y Célia F. Malfatti ${ }^{(1)}$ \\ (1) Universidade Federal do Rio Grande do Sul, Escuela de Ingeniería, Departamento de \\ Metalurgia, Porto Alegre-Brasil (e-mail: celia.malfatti@ufrgs.br) \\ (2) Universidade Feevale, Instituto de Ciencias Exactas y Tecnológicas, Grupo de Investigación \\ en Materiales, Novo Hamburgo, RS-Brasil (e-mail: eduardoluis@feevale.br)
}

Recibido Dic. 06, 2011; Aceptado Feb. 07, 2012; Versión final recibida May. 03, 2012

\section{Resumen}

El objetivo de este trabajo fue estudiar la influencia de los parámetros operacionales del proceso de electropulido de aleaciones de titanio, involucrando la obtención de superficies nanoestructuradas. Se sabe que los parámetros del proceso de electropulido ejercen una importante influencia sobre la formación de materiales nanoestructuradas, promoviendo la obtención de propiedades bien específicas. En este trabajo se realizó el electropulido de la aleación Ti6AI4V lijadas mecánicamente, variando el tiempo de pulido. Luego del electropulido la morfología de la superficie fue evaluada por microscopia de fuerza atómica y la hidrofobicidad fue determinada mediante el ángulo de contacto. Los resultados preliminares muestran que el control sobre el tamaño y la profundidad de las nanoestructuras en esas superficies es posible por la variación del tiempo.

Palabras clave: electropulido, biomateriales, Ti6Al4V, nanoestructuras

\section{Nanostructured Surfaces alloy Ti6AI4V. Influence of Electropolishing Time}

\begin{abstract}
The aim of the present research was to study the influence of operational parameters in the electropolishing process of titanium alloys, involving nanostructured surfaces. The electropolishing operational parameters influence the formation of nanostructured materials, promoting the obtainment of specific properties. In this work, a variation time was studied to produce nanostructured surfaces by the electropolishing process. The surfaces obtained were evaluated by atomic force microscopy and surface hydrophobicity was determined by the measurement of the contact angle. Preliminary results showed that the control on the size and depth of nanostructures on these surfaces is possible by electropolishing time variation.
\end{abstract}




\section{INTRODUCCION}

El titanio y sus aleaciones han sido ampliamente utilizados en implantes ortopédicos, tales como prótesis para sustituir articulaciones, debido, a su elevada dureza, bajo módulo de elasticidad, alta resistencia a la corrosión y excelente biocompatibilidad (Nishiguchi et al., 1999; Isaac et al., 2010; Yong et al., 2009). El titanio presenta alta citocompatibilidad in vitro y en vivo, permitiendo el contacto directo entre hueso-implante (Carvalho et al., 2010). Por lo tanto estos biomateriales no forman compuestos químicos con el hueso, solo quedan incorporados mediante un contacto óseo, donde el metal necesita tener la capacidad de enlazarse al tejido óseo mejorando el injerto de los mismos, otros métodos alternativos están siendo investigados (Isaac et al., 2010).

En las últimas décadas la investigación de biomateriales ha desarrollado superficies innovadoras para implantes buscando una óptima cicatrización del mismo (óseointegración) y se basaron en la respuesta que dan las células osteogénicas respecto, a la adhesión, proliferación y diferenciación celular (Molina et al., 2001; Saldaña et al. 2010; Ravanetti et al., 2010). Debido a su biocompatibilidad y propiedades fisicoquímicas favorables, el titanio se destaca como un substrato ideal para esos tipos de modificaciones (Klein et al., 2010).

Existen muchas investigaciones realizadas que demuestran que la rugosidad de la superficie del implante de titanio afecta la tasa de óseointegración y fijación biomecánica (Le Guéhennec et al., 2007; Velasco et al., 2010). Los cambios de la rugosidad en la superficie también afectan la mojabilidad, siendo que estas determinan el comportamiento de las células debido a la dinámica de absorción de las biomoleculas sobre la superficie (Molina et al., 2001).

La morfología y la rugosidad pueden ser controlados a través de un tratamiento con solución acida o por oxidación, resultando en una topografía a escala nanometrica (Klein et al., 2010; Elías et al., 2008). El tratamiento superficial con ácidos fuertes, como por ejemplo: $\mathrm{HCl}, \mathrm{H}_{2} \mathrm{SO}_{4}, \mathrm{HNO}_{3}$ y $\mathrm{HF}$, es un método empleado para promover la formación de superficies rugosas en implantes (Le Guéhennec et al., 2007). Las alteraciones químicas de la superficie, incluyen, la deposición de hidroxiapatita y la incorporación de iones calcio, fosforo y fluorita (Elías et al., 2008). Por otro lado algunos autores usaron la técnica de anodización en soluciones electrolíticas conteniendo fosfatos para anodizar titanio (Frauchiger et al., 2004) o aluminio (Popat et al., 2005) como métodos alternativos para la obtención de materiales biocompatibles.

La rugosidad de la superficie puede ser clasificada en tres niveles: macrométricas, micrométricas y nanométricas. El nivel macro es definido por las características topográficas a nivel de decenas de micrones hasta milímetros. Esta escala está directamente relacionada con la geometría del implante, rosca del tornillo y tratamientos de superficie macroporosa, obteniéndose una rugosidad mayor de $10 \mu \mathrm{m}$. El nivel micro de los implantes dentales, es definido por la rugosidad de la superficie, encontrándose en el rango de [1-10] $\mu \mathrm{m}$. Varios estudios han demostrado que con este intervalo de rugosidad de la superficie, se obtiene un mayor contacto hueso-implante y una mayor resistencia a la remoción por torque, en comparación a otros tipos de superficie, las superficies a escala nanométrica desempeñan un papel importante en la adsorción de proteínas, adhesión de las células osteoblásticas y en la tasa de óseointegración (Le Guéhennec et al., 2007).

La superficie nanoestructurada adecuada para la óptima adsorción selectiva de proteínas conducentes a la adhesión de células ósteoblásticas aún desconocida (Le Guéhennec et al., 2007). Existen pocos estudios realizados modificando, la rugosidad y la composición química de la superficie a escala nanométrica de una forma reproducible (Le Guéhennec et al., 2007).

El presente trabajo busca estudiar la influencia de la variación del tiempo en el proceso de electropulido, para la obtención de superficies nanoestructuradas de aleaciones de titanio.

\section{MATERIALES Y METODOS}

\section{Preparación superficial}

Se utilizó como substrato la aleación Ti6Al4V (discos de $\varnothing 9,55 \mathrm{~mm}$ ). Como preparación superficial, las muestras fueron lijadas manualmente con lijas de carburo de silicio hasta la lija 600 
(pulimientos con lijas de numeración mayor no influyen significativamente en el electropulimiento (Manhabosco, 2011), luego las muestras fueron electropulidas.

\section{Pulimiento electroquímico}

Para el pulido electroquímico, se utilizó una solución ácida, conteniendo, $\mathrm{HF}$ y $\mathrm{H}_{2} \mathrm{SO}_{4}$, platino como cátodo y la muestra como ánodo, en una fuente generadora (MPC-303DI, Minipa).

Los parámetros operacionales fueron, densidad de corriente de $0,3 \mathrm{~A} / \mathrm{cm}^{2}$, se varió el tiempo en 2 , 4 y 8 minutos (tabla 1), el sistema de electropulido fue mantenido a una temperatura de $8 \pm 1 \stackrel{\circ}{ } \mathrm{C}$, al terminar el proceso, la muestra se colocó en alcohol etílico y se lavó con agua destilada.

Tabla 1. Relación de las muestras y parámetros estudiados

\begin{tabular}{|c|c|c|}
\hline Muestra & Densidad de corriente $\left(\mathrm{A} / \mathrm{cm}^{2}\right)$ & Tiempo (minutos) \\
\hline AC230 & 0,3 & 2 \\
\hline AC430 & 0,3 & 4 \\
\hline AC830 & 0,3 & 8 \\
\hline
\end{tabular}

\section{Caracterización de las muestras electropulidas}

Las muestras electropulidas fueron caracterizadas en lo referente a la morfología y la hidrofobicidad para determinar el ángulo de contacto.

\section{Microscopía de fuerza atómica}

La microscopía de fuerza atómica (AFM) es una importante herramienta para investigar las estructuras nanométricas, permiten ver imágenes en tres dimensiones, en este trabajo esta técnica fue empleada para evaluar la morfología y la rugosidad de las superficies obtenidas, se usó un microscopio de barrido de punta de prueba (Shimadzu SPM-9500J3).

Las imágenes fueron obtenidas por AFM, operando en modo de contacto, con sondas de nitruro de silicio (NANOSENSORS) y scanner con variación vertical de $8 \mu \mathrm{m}$ y un área de barrido de $125 \times 125 \mu \mathrm{m}$. La evaluación de la rugosidad de la superficie en nanómetros fue realizada a través del AFM, se midió, $R_{a}$ (medida de la rugosidad superficial en nanómetros), $R_{z}$ (media absoluta de los cinco picos mayores y cinco menores valles, en nanómetros), según las ecuaciones 1 y 2 (Santos, 2008) y $R_{m s}$ (media de la raíz cuadrada de la rugosidad).

Se obtuvieron 10 imágenes de la superficie en diferentes regiones de la muestra y estos datos se consideran en el cálculo de la desviación estándar de las rugosidades que se presentan en las tablas 2 y 3 . En el análisis de AFM, la distancia de barrido empelado para el estudio de la rugosidad es de $2 \times 2 \mu \mathrm{m}$.

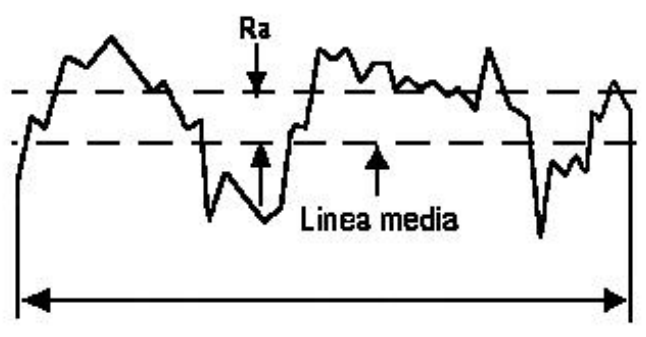

Fig.1: Esquema de la medida de $\mathrm{R}_{\mathrm{a}}$

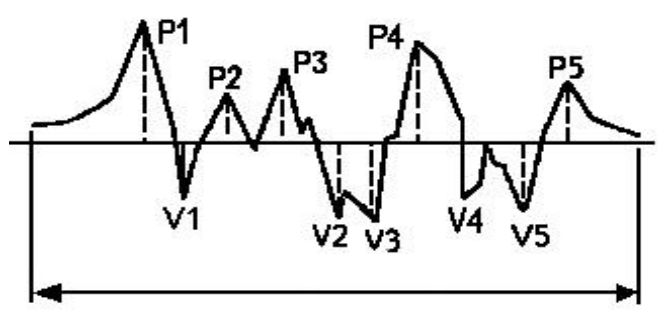

Fig.2: Esquema de la medida de $\mathrm{R}_{\mathrm{z}}$

$R_{\alpha}=\frac{Y_{1}+Y_{2}+Y_{3 \ldots} Y_{N}}{N}$ 
$R_{z}=\frac{\left(P_{1}+P_{2}+\cdots P_{5}\right)+\left(V_{1}+V_{2} \cdots V_{N 5}\right)}{5}$

\section{Perfilometría}

La técnica de perfilometría, es empleada para cuantificar la topografía superficial de un material. Las microestructuras de las superficies fueron evaluadas por un perfilómetro (PRO500 3D) que consiste en una sonda de contacto que se mueve sobre una superficie del material de 250x250 $\mu \mathrm{m}$. La topografía es analizada bidimensional y tridimensionalmente, calculándose las rugosidades $R_{a}, R_{z}$ y $R_{m s}$.

La rugosidad de la superficie puede ser dividida en tres niveles, dependiendo de la escala de los recursos: Topologías macro, micro y nanométricas. El nivel macro topográfico es definido por características topográficas, en el rango de milímetros a decenas de micrones, el perfil micro topográfico es definido por la rugosidad de la superficie en el rango de 1-10 $\mu \mathrm{m}$ y el perfil nanométrico es inferior a $100 \mathrm{~nm}$, los equipos (AFM) permiten la monitorización de la rugosidad hasta 700 nm (Le Guéhennec et al., 2007).

\section{Mojabilidad}

Esta prueba fue realizada por el método de la gota sésil, por un equipo desarrollado por el laboratorio de óptica y laser, del instituto de física de la UFRGS, que determina el ángulo de contacto formado por la gota de la solución PBS (solución salina tampón con fosfato) y el substrato analizado, mediante un programa de análisis de imágenes, desarrollado por el mismo laboratorio. Esta técnica analiza la mayor o menor capacidad hidrofílica del substrato (Wenzel, 1936; Wenzel, 1949)

Cuando una gota líquida es depositada sobre una superficie horizontal, la mojabilidad está relacionada al comportamiento del ángulo de contacto entre la línea tangente a la superficie del líquido y la superficie horizontal. Un ángulo de contacto superior a $90^{\circ}$ caracteriza una superficie con poca mojabilidad (hidrofóbica), cuando el ángulo de contacto es menor de $60^{\circ}$ caracteriza una superficie hidrofílica (Santos, 2008).

La forma de la gota depositada sobre una superficie es determinada por la interacción de tres fuerzas aplicadas en la línea de contacto, formada por cada par entre las tres fases: La línea entre el sólido y la fase líquida (YsL), entre el sólido y la fase de vapor (Ysv) y entre el líquido y la fase de vapor (Y Y LV). Las tres fuerzas (fig. 3) definen la forma global de la gota, siendo, $Y$, la tensión superficial (energía por unidad de superficie). (Michels, 2008).

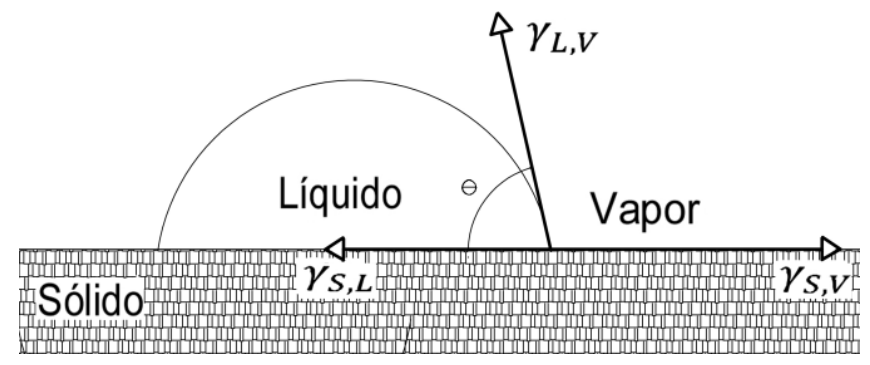

Fig. 3: Fuerzas actuantes en una gota depositada sobre una superficie sólida

El ángulo de contacto $(\Theta)$ formado entre (Ysv) y (YLV), es obtenido por la ecuación de Young y Dupré, en la condición de equilibrio:

$\cos \theta=\frac{\gamma_{S V}-\gamma_{S L}}{\gamma_{L V}}$ 
Las superficies donde el ángulo sea mayor que $90^{\circ}$ y menor de $150^{\circ}$, son clasificados como hidrofóbicas y mayores de $150^{\circ}$ son considerados super hidrofóbicas (Michels, 2008).

\section{RESULTADOS Y DISCUSIONES}

\section{Morfología y rugosidad}

Las muestras electropulidas (Ti6Al4V) se evaluaron morfológicamente, la figura 4, muestra la topografía de la superficie y la figura 5, presenta la formación de las nanoestructuras, se puede observar que los parámetros empleados para el proceso de electropulido permiten obtener una buena superficie nanoestructurada. En las figuras mencionadas, se verifica que el incremento del tiempo de electropulido generó un aumento en la dimensión de la superficie nanoestructurada resultando en estructuras de mayor profundidad. Ocurre la formación de una capa porosa cuya estructura de poros es razonablemente ordenada, variando el diámetro de los poros del orden de decenas de $\mathrm{nm}$, los poros se orientan perpendicularmente a la superficie metálica y sus paredes forman una estructura hexagonal (forma de colmena de abeja) (Muller, 2008). Además, no hubo variación significativa en los valores de la nanorugosidad (tabla 2) y existe una tendencia al aumento de la microrugosidad con el tiempo de electropulido (tabla 3).
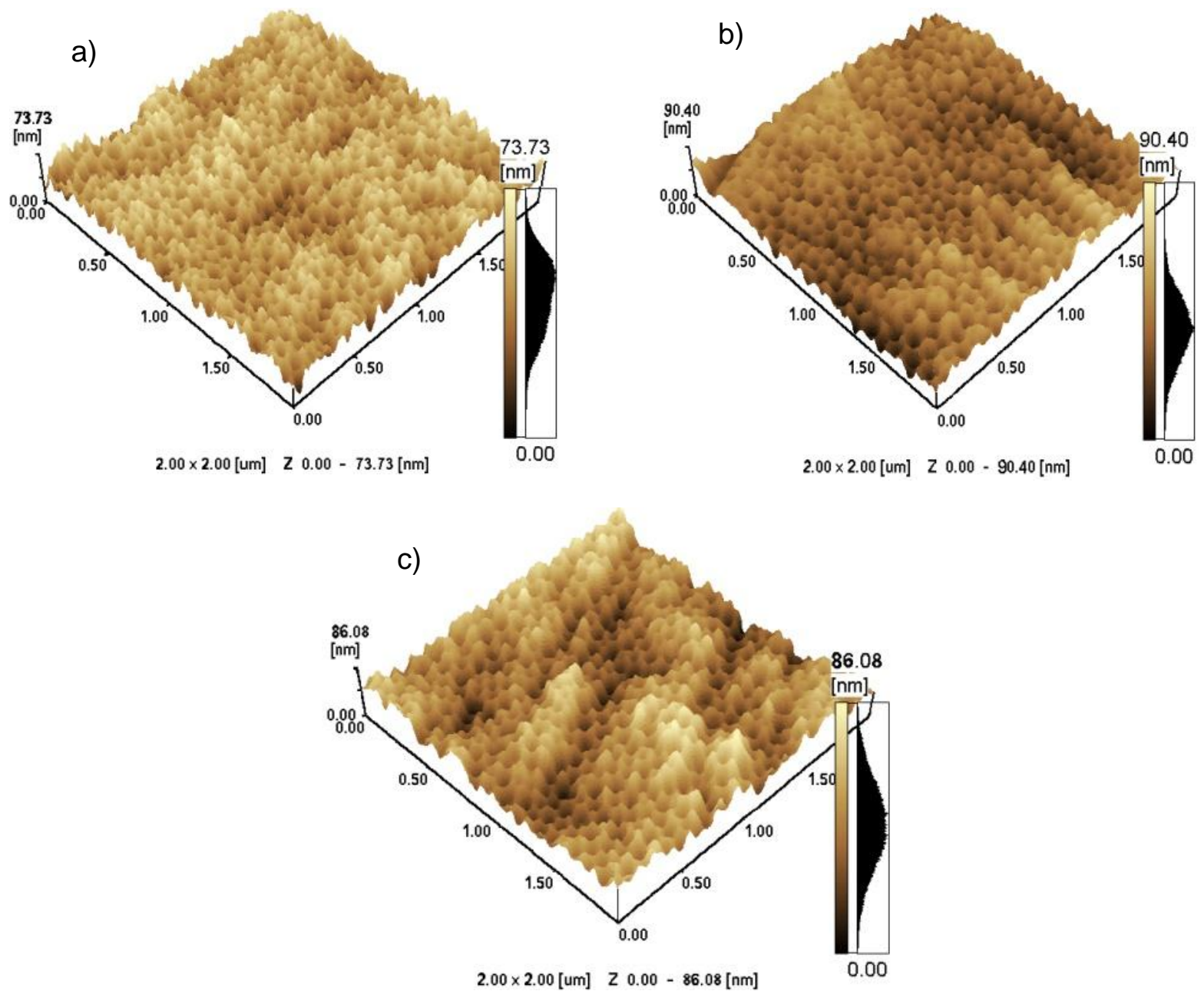

Fig. 4: Imágenes tridimensionales de los substratos luego del electropulido: (a) AC230, (b) AC430, (c) AC830

Durante el proceso de eletropulido ocurre el efecto de polarización de la muestra y depende de la densidad de corriente, tiempo del proceso y la temperatura. En el presente trabajo se evaluó, solo el efecto del tiempo de electropulido, para una densidad de corriente de $0,30 \mathrm{~A} / \mathrm{cm}^{2}$. El substrato después de haber sido lijado con lija 600 , poseía una rugosidad $R_{a}=0,07 \mu \mathrm{m}$ y con el incremento 
del tiempo de electropulimiento hay una tendencia al aumento de la microrugosidad $(\mathrm{Ra}=0,11$ $\mu \mathrm{m}$ ), debido a que el material es una aleación, la disolución puede ser favorecida en algunas micro regiones, por la formación de micro pares galvánicos (Barranco et al., 2007).

Según investigaciones realizadas (Isaac et al., 2010) la rugosidad nanometrica observado es aproximadamente $10 \mathrm{~nm}$, estas mencionan que cuanto mayor es la rugosidad de la superficie mayor será el proceso de óseointegración. Los perfiles de superficie en escala nanométrica desempeñan un papel importante en la adsorción de proteínas, adhesión de células osteoblásticas y en la tasa de óseointegración, en cuanto a la rugosidad micrométrica, favorece el contacto hueso-implante y promueve una mayor resistencia a la remoción por torque. Los resultados obtenidos muestran que el control del tiempo de electropulido puede influenciar directamente sobre la propiedad superficial del implante (Le Guéhennec et al 2007; Barranco et al, 2010; Barranco et al, 2011).
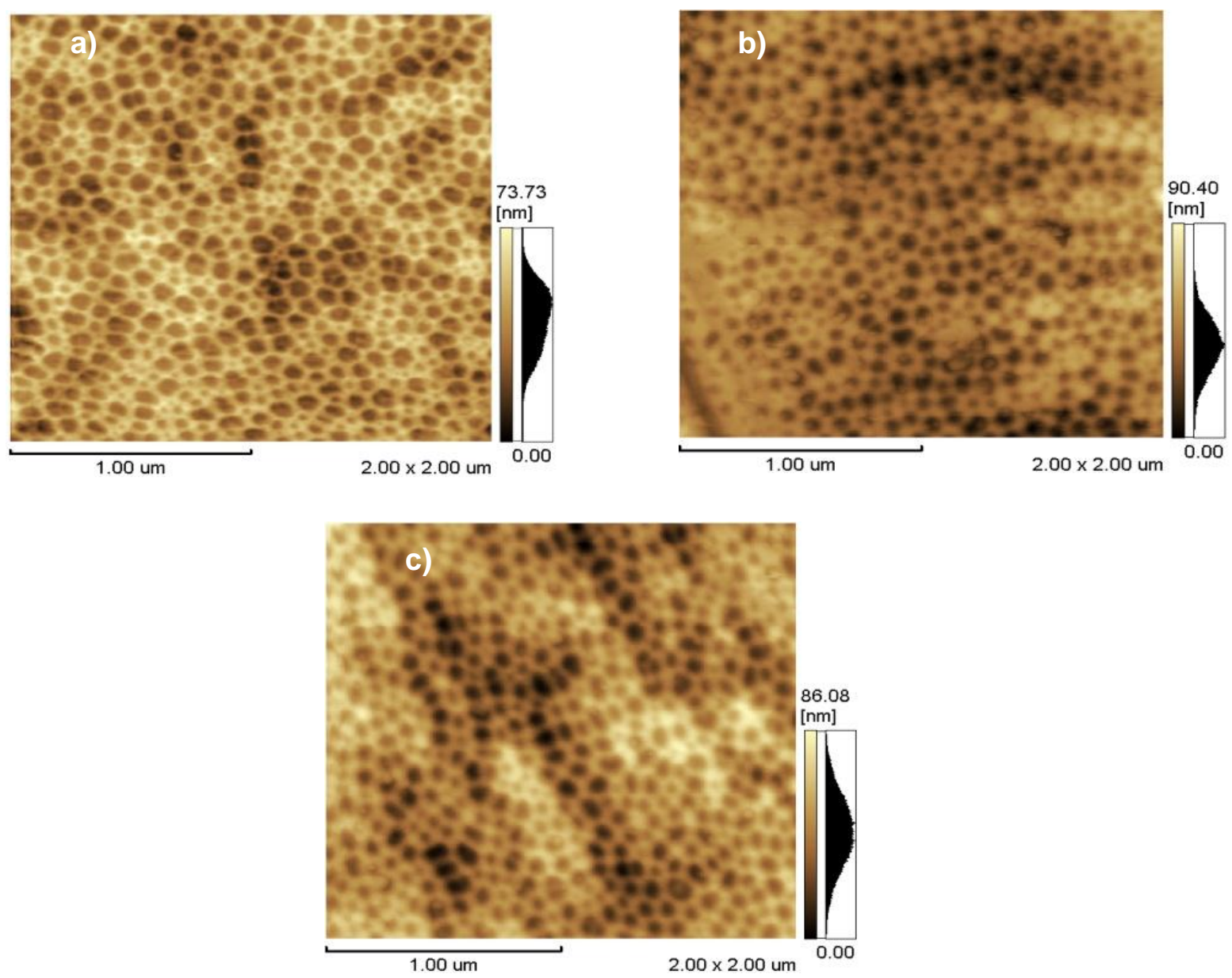

Fig. 5: Imágenes bidimensionales de los substratos luego del electropulido: (a) AC230, (b) AC430, (c) AC830

Tabla 2. Medidas de nanorugosidad

\begin{tabular}{|c|c|c|c|}
\hline \multirow{2}{*}{ Muestra } & \multicolumn{3}{|c|}{ Rugosidad } \\
\cline { 2 - 4 } & $\mathrm{Ra}(\mathrm{nm})$ & $\mathrm{Rms}(\mathrm{nm})$ & $\mathrm{Rz}(\mathrm{nm})$ \\
\hline AC230 & $9,54 \pm 0,21$ & $12,07 \pm 0,26$ & $44,43 \pm 1,32$ \\
\hline AC430 & $8,43 \pm 0,23$ & $10,50 \pm 0,34$ & $49,89 \pm 3,26$ \\
\hline AC830 & $8,88 \pm 0,22$ & $11,06 \pm 0,29$ & $41,23 \pm 2,45$ \\
\hline
\end{tabular}


Tabla 3. Medidas de microrugosidad

\begin{tabular}{|c|c|c|c|}
\hline \multirow{2}{*}{ Muestra } & \multicolumn{3}{|c|}{ Rugosidad } \\
\cline { 2 - 4 } & $\mathrm{Ra}(\mu \mathrm{m})$ & $\mathrm{Rms}(\mu \mathrm{m})$ & $\mathrm{Rz}(\mu \mathrm{m})$ \\
\hline AC230 & $0,04 \pm 0,01$ & $0,04 \pm 0,01$ & $0,25 \pm 0,03$ \\
\hline AC430 & $0,07 \pm 0,03$ & $0,09 \pm 0,02$ & $0,50 \pm 0,22$ \\
\hline AC830 & $0,11 \pm 0,02$ & $0,13 \pm 0,03$ & $0,65 \pm 0,09$ \\
\hline
\end{tabular}

\section{Mojabilidad}

Se puede observar (tabla 4 y figura 6) que todas las superficies obtenidas presentan un carácter hidrofílico (ángulo de contacto menor de 90), el aumento del tiempo de electropulido promovió la disminución del valor del ángulo de contacto medio. Ese comportamiento indica la influencia de la microrugosidad sobre la mojabilidad de la superficie, ya que la nanorugosidad prácticamente no sufrió modificación con el aumento del tiempo de electropulido.
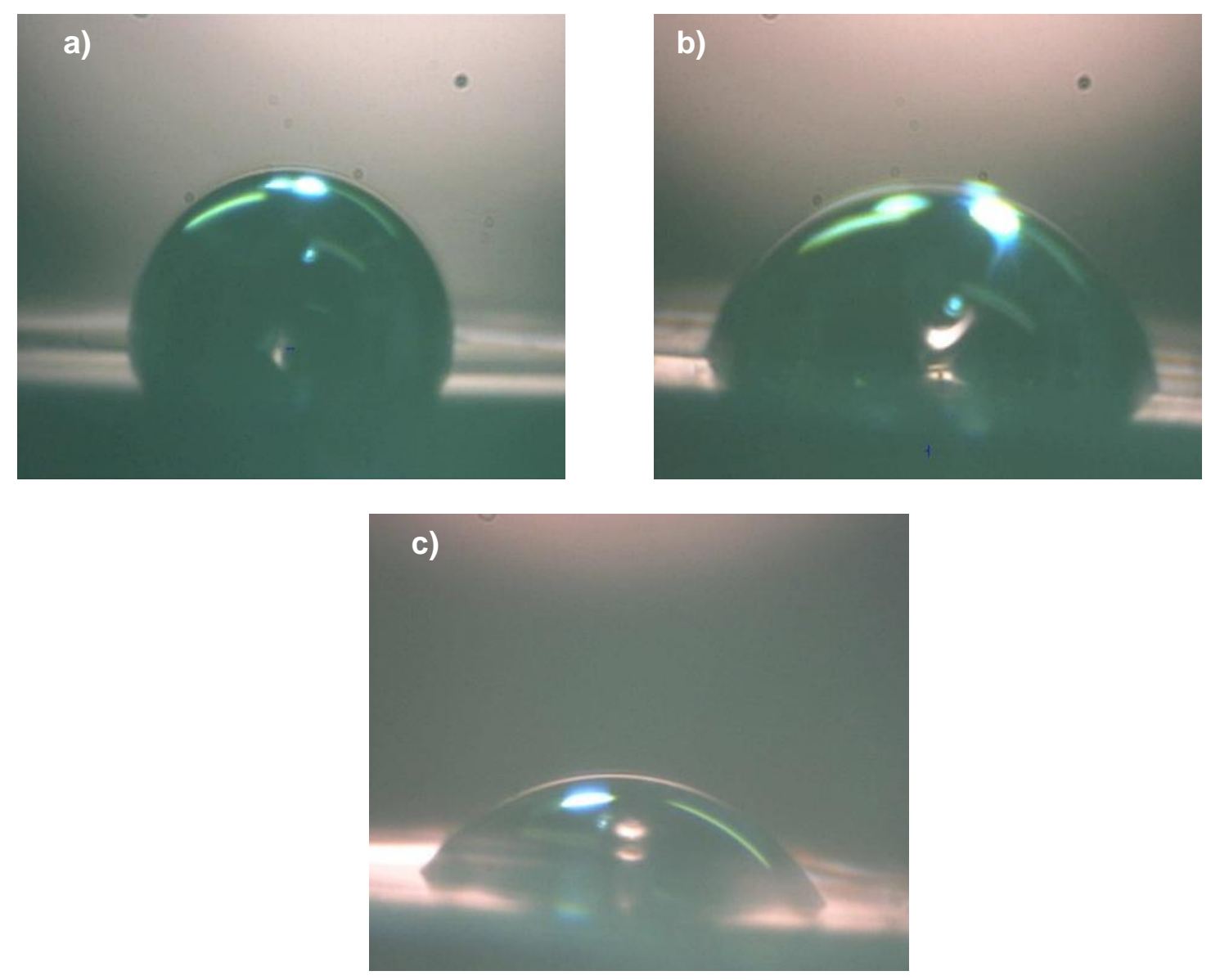

Fig. 6: Imágenes de mojabilidad con PBS (a) AC230, (b) AC430, (c) AC830

Tabla 4. Ángulo de contacto medio y desviación estándar

\begin{tabular}{|c|c|c|}
\hline Muestra & $\begin{array}{c}\text { Ángulo de } \\
\text { contacto }\end{array}$ & $\begin{array}{c}\text { Desviación } \\
\text { estándar }\end{array}$ \\
\hline AC230 & $85,65^{\circ}$ & $\pm 1,79$ \\
\hline AC430 & $72,04^{\circ}$ & $\pm 3,38$ \\
\hline AC830 & $53,27^{\circ}$ & $\pm 3,68$ \\
\hline
\end{tabular}




\section{CONCLUSIONES}

EL electropulido fue utilizado para obtener superficies nanoestructuradas de acabado brillante a través del proceso electroquímico. El mecanismo involucra la formación de un oxido intermedio, que fue el responsable para garantizar un ataque uniforme en la superficie.

A partir del análisis de la morfología, se verificó la formación de superficies nanoestructuradas, las cuales con el aumento del tiempo de electropulido presentan un aumento de la microrugosidad, casi sin afectar la nanorugosidad superficial.

De las imágenes obtenidas por AFM (figura 4 y 5), se observa que el pulimiento de la superficie es bastante regular, pero con una topografía diferenciada entre sí (tablas 2 y 3 ) y se verifica por los valores de los parámetros $R_{a}, R_{z}$ y $R_{m s}$, tanto a escala nanométrica y micrométrica. El aumento de la microrugosidad generó una mayor mojabilidad de la superficie (menor ángulo de contacto medio).

La principal contribución de este trabajo, es demostrar la influencia del tiempo, en la morfología de la nanoestructura, considerando que el objetivo de la aplicación de este material es el área biomédica.

\section{AGRADECIMIENTOS}

El presente trabajo fue realizado con el apoyo de la CAPES, que es la entidad del gobierno Brasilero orientado a la formación de recursos humanos, CNPq, Laboratorio de mecánica de la universidad Feevale y al Laboratorio de Investigaciones de Corrosión de la UFRGS (LAPEC).

\section{REFERENCIA}

Barranco, V., M. L. Escudero y M. C. García-Alonso, 3D, chemical and electrochemical characterization of blasted Ti6Al4V surfaces: Its influence on the corrosion behavior, Electrochimica Acta, 52, 4374-4384 (2007).

Barranco, V., M. L. Escudero y M. C. García-Alonso, Characterization of roughness and pitting corrosion of surfaces modified by blasting and thermal oxidation. Surface and Coatings Technology, 204, 3783-3793 (2010).

Barranco, V., M. L. Escudero y M. C. García-Alonso, Influence of the microstructure and topography on the barrier properties of oxide scales generated and blasted Ti6Al4V surfaces. Acta Biomaterialia, 7, 2716-2725 (2011).

Carvalho, D. R. y otros cinco autores, Characterization and in vitro cytocompatibility of an acidetched titanium surface, Brazilian Dental Journal: 21(1), 3-11 (2010).

Elias, C. N., Y. Oshida, J. H. C. Lima y C. A. Muller, Relationship between surface properties (roughness, wettability and morphology) of titanium and dental implant removal torque, Journal of the Mechanical Behavior of Biomedical Materials, 1, 234-242 (2008).

Frauchiger, V. M., F. Schlottig, B. Gasser y M. Textor, Anodic plasma-chemical treatment of CP titanium surfaces for biomedical application, Biomaterials, 25 (4): 593 (2004).

Isaac, J. y otros cinco autores, Bioengineered titanium surfaces affect the gene-expression and phenotypic response of osteoprogenitor cells derived from mouse calvarial bones, European Cells and Materials, 20, 178-196 (2010).

Klein, M. O. y otros seis autores, Long-term response of osteogenic cells on micron and submicron-scale-structure hydrophilic titanium surfaces: sequence of cell proliferation and cell differentiation. Clinical Oral Implants Research, 21, 642-649 (2010). 
Le Guéhennec, L., A. Soueidan, P, Layrolle y Y. Amouriq, Surface treatments of titanium dental implants for rapid osseointegration. Dental Materials, 23, 844-854 (2007).

Manhabosco, T. M., S. M. Tamborin, C. B. dos Santos y I. L. Muller, Tribological, electrochemical and tribo-electrochemical characterization of bare and nitrided Ti6Al4V in simulated body fluid solution, Corrosion Science, 53, 1786-1793 (2011).

Michels, A. F., D. E. Weibel, P. L. G. Jardim y F. Horowitz, Tópicos em Nanociência e Nanotecnologia, pp 68, Editoral UFRGS, Porto Alegre, Brasil (2008).

Molina, M. P. y otros siete autores, Role of wettability and nanoroughness on interactions between osteoblast and modified silicon surfaces. Acta Biomaterialia, 7, 771-778 (2011).

Muller, I. L. Tópicos em Nanociência e Nanotecnologia, pp 68, Editoral UFRGS, Porto Alegre, Brasil (2008).

Nishiguchi, S. y otros cinco autores. The effect of heat treatment on bone-bonding ability of alkalitreated titanium, Biomaterials, 20, 491-500 (1999).

Popat, K. C. y otros seis autores. Influence of nanoporous alumina membranes on long-term osteoblast response, Biomaterials, 26 (22): 4516 (2005).

Ravanetti, F. y otros seis autores, In vitro cellular response and in vivo primary oesteointegration of electrochemically modified titanium, Acta Biomaterialia, 6, 1014-1024 (2010).

Rupp, F. y otros cuatro autores, Roughness induced dynamic changes of wettability of acid etched titanium implant modifications, Biomaterials, 25, 1429-1438 (2004).

Saldaña, L. y N. Vilaboa, Effects of micrometric titanium particles on osteoblast attachment and cytoskeleton architecture, Acta Biomaterialia, 6, 1649-1660 (2010).

Santos, A. R, Efeito da oxidação anódica de titânio comercialmente puro revestido ou não com fibronectina na interface osteoblastos humanos-superfície de titânio, Tesis de doctorado, Dpto. Eng. Metalúrgica e de Materiais, UFRJ, Rio de Janeiro, Brasil (2008).

Velasco, E. y otros cuatro autores, In vitro evaluation of cytotoxicity and genotoxicity of a commercial titanium alloy for dental implantology, Mutation Research, 702, 17-23 (2010).

Wenzel, R. N, Resistance of solid surfaces wetting by water, Industrial \& Engineering Chemistry, 28, 988-994 (1936).

Wenzel, R. N, Surface roughness and contact angle, The Journal of Physical Chemistry, 53,1466 1467 (1949).

Yong. L., G. S. Rong y Z. M. Jin, Wettability modification for biosurface of titanium alloy by means of sequential carburization, Journal of Bionic Engineering, 6, 219-223 (2009). 
